\title{
DISCUSSION
}

\section{Analysis of single-fluid jet grouting}

\author{
P. CROCE and A. FLORA (2000). Géotechnique 50, No. 6, 739-748
}

\section{A. Greenwood,}

I congratulate Professor Croce and his colleague on tackling the analysis of the jet grouting process. This topic is ripe for study.

Concerning the diameter of columns resulting from jet erosion, the key question is the correlation of soil properties derived from site investigation with diameter. The need for initial field testing to confirm design assumptions based on empirical knowledge is often prohibitively expensive.

The correlation with penetration tests and undrained shear strengths is poor. In gravelly soils the scatter of penetration resistance is large, and averages are unrepresentative of the resistance to the physical effects of jetting out the matrix of finer soils. In cohesive soils surely plasticity index is a significant guide in conjunction with undrained strength?

Regarding Fig. 10 of the paper relating diameter to energy input, the linear relation shown applies only over a small range of energies. The Japanese have demonstrated columns of 5-7 m diameter using energy values 6-10 times those of the range indicated. The loss of energy at large diameters must result in a declining relationship.

These large diameters have been achieved using single-jet techniques. The air used in double or triple systems loses its apparent effects at such a range except for airlifting effluent from the bore.

Moreover, the large diameters are achieved using jet pressures the same as those in first-generation machines with increased volumes of jet fluids. This confirms that momentum rather than pressure is the key to erosion.

On the first ever jet grouting contract in 1962 for an underground sewage pumping station at East Pentire, Newquay, UK, Cementation used a single grout jet at 10 bar pressure with an $8 \mathrm{~mm}$ diameter orifice. Column sizes of $800 \mathrm{~mm}$ were achieved in a uniform medium gravel with about SPT $N=20$. The effluent was collected, hydrocycloned to remove coarse particles and recirculated with added cement until the correct quantity was achieved. The benefits in terms of fewer environmental and disposal problems and less waste of cement are obvious. This process used about half the cement used with current techniques. It is astonishing that these practices are not used today.

Concerning the control of the soilcrete composition, this is constrained by the necessity of having to compromise with the jetting requirements. It is essential that the jet be sufficiently fluid, and that it has sufficient momentum to be effective. This limits grout material proportions. When separate air and water jets are employed, the ratios of material volumes are altered further.

Using a material input/output balance as illustrated by the authors in the appendix to the paper it is also important to know the cavity volume (and hence its diameter) accurately, as otherwise significant errors arise in the material proportions calculated for the soilcrete.

Furthermore, when jetting in soils containing coarse sands and gravels there may be insufficient velocity in effluent rising up the annular space around the drill pipes to raise the coarser particles. They necessarily remain in the bore, sinking by hindered settlement in the soilcrete slurry. Hence they do not take part in the fluid circulation and should be excluded from the balance.

Control can sometimes be exercised on the particle sizes remaining in circulation, depending on the particle size distribution of the soil. By drilling (and possibly temporarily casing) a borehole of a chosen diameter the up-flow velocity can be predetermined. By reference to the particle size distribution the proportions retained and settling can be controlled to some extent by choosing an appropriate velocity and hence hole diameter. Regard must be taken of the lifting capacity rendered by the rheological properties of the fairly thick effluent (which is essentially the same as the in-situ soilcrete without the heavier particles).

\section{A. L. Bell, Keller Ground Engineering}

In his presentation, Professor Croce provides an alternative energy analysis for single-fluid jet grouting, and he rightly draws attention to the need to deal with the energy at the nozzle rather than the pump. Development at Keller Ground Engineering during the last 10 years has, among other things, been directed at improving the hydraulic efficiency of the jet grout monitor. The diameters, particularly for clay soils, reported in the 1993 review to which Professor Croce refers are therefore somewhat conservative in the light of current experience.

My question relates to the energy analysis, which has been developed for the single-jet system. Clearly the method would have a much broader application if applied to the other methods. I should be interested in Professor Croce's comments on the applicability to the triple-jet system.

\section{Authors' reply}

The authors would like to thank the writers for their discussions, and for the opportunity to clarify some of the points raised in the paper.

The phenomena produced by jet grouting are very complex, and the degree of complexity increases with the number of fluids injected into the subsoil. The authors have thus chosen to tackle the single-fluid system as the first step of a broad research project aiming to provide rational design methods for all jet grouting systems.

The analysis of single-fluid jet grouting presented in the paper was based mainly on the experimental evidence acquired from a relatively small number of cases, where it was possible to perform accurate observations and measurements. Therefore the reported range of column diameters is restricted to the relevant energy values. For increasing energy, the diameter should correspondingly increase, as confirmed by the Japanese results mentioned by Dr Greenwood.

However, the relationship between jet energy and column diameter should be further investigated, and, by collecting a large number of field data, empirical design charts may eventually be produced. These charts should also account for the relevant soil properties: permeability for gravels, shear strength for finer soils. In this respect, Fig. 10 should not be considered as a design chart, although it shows the influence of jet energy and soil grading. General correlations may be attempted in the future by using the results of proper in-situ tests (e.g. borehole seepage tests for gravels, SPT for sands, CPT for clays).

Of course technological developments must be accounted for, and it seems logical that recent improvements in the hydraulic efficiency should result in larger column diameters, as reported by $\mathrm{Dr}$ Bell. However, this finding is consistent with the energy expression proposed by the authors, since higher hydraulic efficiency just results in larger values of the kinetic energy at the nozzles. It is also observed that jet grouting effectiveness has recently increased not only because of lower hydraulic 
losses in the system, but also because of new monitor configurations obtained mainly by inclining the nozzles.

The authors believe that a thorough understanding of jet grouting mechanisms can be guided by theoretical modelling of grout-soil interaction. To this aim, it seems feasible to develop two different models, for soil permeation and for soil erosion respectively. In particular, for modelling soil permeation, turbulent flow regime and grout viscosity should be accounted for, and, for the analysis of soil erosion, drained and undrained shear strength should respectively be considered. In fine-grained soils, plasticity index may also play a role as suggested by $\mathrm{Dr}$ Greenwood, as far as it affects undrained shear strength.

With regard to double- and triple-fluid systems it is well known that when the disrupting jet (respectively grout or water) is surrounded by compressed air, the energy losses are substantially reduced. Therefore higher diameters are usually obtained, although the air effect fades out as the diameter gets larger, as suggested by Dr Greenwood. It follows that the energy approach presented for single-fluid systems should also apply to doubleand triple-fluid systems, as proposed by Dr Bell. In particular, the jet energy at the nozzles could still be calculated by equation (3), applied to the relevant grout or water jet, but different correlations are expected from experimental data. By comparing the correlations pertaining to different systems, for each soil type, it might be possible to evaluate their efficiency. Such evaluation could be aided by theoretical calculation of the energy dissipation that takes place in the ground.

Finally Dr Greenwood has pointed out some problems that may be encountered in the interpretation of experimental measurements by input/output balance. In fact very careful measurements are needed, and the proposed method is best suited for research. The method can be particularly useful, however, for investigating jet grouting effects on fine sands and silty sands, where direct observation of soilcrete structure is not feasible. 